\title{
Prediction of individuals at high risk of chronic kidney disease during treatment with lithium for bipolar disorder
}

Joseph F. Hayes 1,2* David P. J. Osborn 1,2, Emma Francis ${ }^{1}$, Gareth Ambler ${ }^{3}$, Laurie A. Tomlinson4, Magnus Boman ${ }^{5,6}$, Ian C. K. Wong ${ }^{7,8}$, John R. Geddes ${ }^{9}$, Christina Dalman ${ }^{10}$ and Glyn Lewis ${ }^{1,2}$

\begin{abstract}
Background: Lithium is the most effective treatment in bipolar disorder. Its use is limited by concerns about risk of chronic kidney disease (CKD). We aimed to develop a model to predict risk of CKD following lithium treatment initiation, by identifying individuals with a high-risk trajectory of kidney function.

Methods: We used United Kingdom Clinical Practice Research Datalink (CPRD) electronic health records (EHRs) from 2000 to 2018. CPRD Aurum for prediction model development and CPRD Gold for external validation. We used elastic net regularised regression to generate a prediction model from potential features. We performed discrimination and calibration assessments in an external validation data set.

We included all patients aged $\geq 16$ with bipolar disorder prescribed lithium. To be included patients had to have $\geq$ 1 year of follow-up before lithium initiation, $\geq 3$ estimated glomerular filtration rate (eGFR) measures after lithium initiation (to be able to determine a trajectory) and a normal ( $\left.\geq 60 \mathrm{~mL} / \mathrm{min} / 1.73 \mathrm{~m}^{2}\right)$ eGFR at lithium initiation (baseline). In the Aurum development cohort, 1609 fulfilled these criteria. The Gold external validation cohort included 934 patients.

We included 44 potential baseline features in the prediction model, including sociodemographic, mental and physical health and drug treatment characteristics. We compared a full model with the 3-variable 5-year kidney failure risk equation (KFRE) and a 3-variable elastic net model.

We used group-based trajectory modelling to identify latent trajectory groups for eGFR. We were interested in the group with deteriorating kidney function (the high-risk group).
\end{abstract}

Results: The high risk of deteriorating eGFR group included 191 (11.87\%) of the Aurum cohort and 137 (14.67\%) of the Gold cohort. Of these, 168 (87.96\%) and 117 (85.40\%) respectively developed CKD 3a or more severe during follow-up. The model, developed in Aurum, had a ROC area of 0.879 (95\% $\mathrm{Cl} 0.853-0.904)$ in the Gold external validation data set. At the empirical optimal cut-point defined in the development dataset, the model had a sensitivity of 0.91 (95\%Cl 0.84-0.97) and a specificity of 0.74 (95\% Cl 0.67-0.82). However, a 3-variable elastic net model (including only age, sex and baseline eGFR) performed similarly well (ROC area $0.888 ; 95 \% \mathrm{Cl} 0.864-0.912$ ), as did the KFRE (ROC area 0.870; 95\% Cl 0.841-0.898).

(Continued on next page)

\footnotetext{
* Correspondence: joseph.hayes@ucl.ac.uk

'Division of Psychiatry, UCL, London, UK

${ }^{2}$ Camden and Islington NHS Foundation Trust, London, UK

Full list of author information is available at the end of the article
}

C C The Author(s). 2021 Open Access This article is licensed under a Creative Commons Attribution 4.0 International License, which permits use, sharing, adaptation, distribution and reproduction in any medium or format, as long as you give appropriate credit to the original author(s) and the source, provide a link to the Creative Commons licence, and indicate if changes were made. The images or other third party material in this article are included in the article's Creative Commons licence, unless indicated otherwise in a credit line to the material. If material is not included in the article's Creative Commons licence and your intended use is not permitted by statutory regulation or exceeds the permitted use, you will need to obtain permission directly from the copyright holder. To view a copy of this licence, visit http://creativecommons.org/licenses/by/4.0/. The Creative Commons Public Domain Dedication waiver (http://creativecommons.org/publicdomain/zero/1.0/) applies to the data made available in this article, unless otherwise stated in a credit line to the data. 
(Continued from previous page)

Conclusions: Individuals at high risk of a poor eGFR trajectory can be identified before initiation of lithium treatment by a simple equation including age, sex and baseline eGFR. Risk was increased in individuals who were younger at commencement of lithium, female and had a lower baseline eGFR. We did not identify strong predicters of eGFR decline specific to lithium-treated patients. Notably, lithium duration and toxicity were not associated with high-risk trajectory.

\section{Background}

Lithium is the most effective maintenance treatment for bipolar disorder and is first-line in all international clinical practice guidelines [1]. However, its use has been declining globally [2]. Reasons for this include the required monitoring due to its narrow therapeutic window and concerns about adverse effects, particularly irreversible kidney failure [3]. In fact, kidney failure is rare [4], with end-stage kidney failure occurring at similar rates to those treated with other mood stabilisers [5], but more commonly than the general population $(0.23 \%$ vs $0.11 \%$ [6]). Bipolar disorder itself appears to be associated with increased risk of kidney failure independent of lithium exposure [7]. There are inconsistencies in the existing literature about the association between kidney failure and lithium treatment duration and episodes of lithium toxicity [8].

Being able to identify individuals at risk of compromised kidney function would have high clinical utility; it would encourage the use of this effective treatment in those at low-risk and so improve outcomes for people with bipolar disorder. In the general population, established risk factors for CKD include age, sex (increased in women), ethnicity (increased in Black, Asian and Minority Ethnic (BAME) populations), family history of kidney disease, smoking, obesity, hypertension, diabetes mellitus, excessive alcohol consumption and acute kidney injury [9]. Prediction models have been developed for end-stage kidney failure in groups with a range of underlying risk [10-14]. These tend to include a small number of core features including age, gender, ethnicity, eGFR and albuminuria. Models then vary in terms of additional features such as glucose, blood pressure, haemoglobin, lipids, calcium and phosphate. It is unclear if features related to mental health are useful in predicting CKD risk at the point of lithium initiation. It is also likely that risk factors for CKD cluster differently in patients with bipolar disorder prescribed lithium [8] so we cannot assume that risk prediction models for CKD that are of value in the general population would apply to people with bipolar disorder receiving lithium. Because CKD requiring clinical intervention (CKD stage 4 or more severe - eGFR $<30 \mathrm{~mL} / \mathrm{min} / 1.73 \mathrm{~m}^{2}$ ) is a rare and late-stage outcome we aimed to develop a model to classify individuals into high-risk and low-risk trajectories of kidney function following lithium treatment initiation.

\section{Methods \\ Population}

This study used patient data from the Clinical Practice Research Datalink (CPRD) Gold and Aurum databases between 1 January 2000 and 31 December 2018. CPRD contains electronic health records (EHRs) from general practices across the UK. Combined, these databases include 42 million patient records from over 1800 primary care practices (www.cprd.com). Both databases contain coded and anonymised data including demographic details, symptoms, diagnoses, prescribed medication, laboratory tests and referrals. CPRD Gold contains data contributed by practices using Vision software and CPRD Aurum contains data contributed by practices using EMIS Web software $[15,16]$. Contributing practices have different geographical distributions; CPRD Gold contains patients from the whole of the UK, whereas Aurum contains only practices from England and Northern Ireland. Therefore, there are some differences in population structures. We used data from the Aurum database for the development of our prediction model and data from the Gold database for external validation. Ethical approval for this study was obtained from the Independent Scientific Advisory Committee of CPRD (protocol no. 18_316). Informed consent was waived because data are anonymised for research purposes. In line with ethical guidance subgroups containing fewer than 5 people are censored in the results section.

\section{Cohort definition}

The cohort comprised any patient who; was aged 16 or over, ever received a diagnosis of bipolar disorder in their clinical record, was prescribed lithium (defined as receiving two or more concurrent prescriptions), had at least a year of follow-up before their first lithium prescription and no previous record of being prescribed lithium (to capture patients' first exposure to lithium), had at least three estimated glomerular filtration rate (eGFR) measures after lithium initiation and had a baseline measure of eGFR $\geq 60 \mathrm{~mL} / \mathrm{min} / 1.73 \mathrm{~m}^{2}$ before starting lithium (normal or close to normal kidney function).

\section{Kidney function trajectories}

eGFR values were calculated from recorded creatinine blood tests using the CKD-EPI eq. [17]. Using the eGFR, 
and the date the blood test was performed relative to lithium initiation, we conducted group-based trajectory modelling to identify latent subgroups within the cohort [18]. We included lithium exposure as a time-varying covariate, as rate of change in eGFR may potentially differ between the lithium exposed period and following lithium cessation. In the process of determining the number of trajectory groups, we initially used a cubic polynomial function for all groups. The final number of groups was determined based on the Bayesian Information Criterion (BIC), trajectory shapes for similarity, and the proportion of cohort members in each class [19]. We initially set a 2-group model and increased the number of groups until BIC was minimised, but no group was less than $10 \%$ of the total cohort. After identifying the optimal number of groups, the level of the polynomial function for each group was reduced from cubic to zero-order until the BIC was minimised. With this final model, each participant was assigned to one of the subgroups based on maximum posterior probability. We were primarily interested in the group predicted to have the most rapidly declining eGFR trajectory; referred to as the high-risk group.

\section{Prediction model features}

We identified features present in a patient's record before they commenced lithium treatment as potential predictors of being in the high-risk group. These included predictors of eGFR decline in the general population and features related to mental health and its treatment that have been previously identified [20] (code lists available on request):

\section{Sociodemographics}

Age, sex, ethnicity (as BAME vs White), relationship status (single vs. in a relationship).

\section{Mental health characteristics}

Illness duration before lithium initiation, presentations for depression, mania, anxiety (including diagnosis and symptoms of generalised anxiety, phobic anxiety and obsessive-compulsive disorder), psychosis (including affective and non-affective psychotic episodes), stress (including adjustment disorders and symptoms of stress), self-harm (including intentional overdose and non-accidental self-injury), disturbed sleep (including insomnias and hypersomias).

\section{Physical health characteristics}

Hyper/hypocalcaemia, hypo/hyperthyroidism, high LDL cholesterol, low HDL cholesterol, hypertension, coronary heart disease, a measure of eGFR $<60 \mathrm{~mL} / \mathrm{min} / 1.73 \mathrm{~m}^{2}$ any time before lithium initiation, type II diabetes mellitus, asthma, weight loss, peptic ulcer, iron deficiency anaemia, liver disease, chronic pulmonary disease, and neurological disorders.

\section{Health behaviours}

Smoking status (never smoked, current smoker, exsmoker), body mass index group (underweight, healthy weight, overweight, obese), cannabis use, other substance misuse, alcohol misuse.

\section{Other drug treatment}

Antipsychotic prescription, other mood stabiliser prescription, antidepressant prescription.

\section{Interactions}

Baseline eGFR with sex and age, sex with age and body mass index group.

\section{Statistical analysis}

We described differences in prevalence of binary covariates and medians of continuous covariates between high-risk and low-risk groups using $p$ values from chisquared tests. We used probit elastic net regression with 10 -fold cross-validation to perform variable selection and penalization of coefficients to generate the prediction model in the Aurum cohort. Elastic net is a regularisation method for regression and classification models which comprises the Least Absolute Shrinkage And Selection Operator (LASSO) penalty (L1) and the ridge penalty (L2) [21]. The LASSO (L1) penalty function performs variable selection and dimension reduction by shrinking coefficients, whilst the ridge (L2) penalty function shrinks the coefficients of correlated variables toward their average. The overall elastic net is a function of parameters $\lambda$ and $\alpha(0 \leq \alpha \leq 1)$, with $\lambda$ being a parameter for the level of penalty, whilst $\alpha$ being the weight of L1 penalty and (1- $\alpha)$ being that of L2 penalty function. We reported receiver operating characteristic (ROC) area (and 95\% confidence interval) (CI), sensitivity and specificity at the empirical optimal cut-off point using Youden's index and the predictive accuracy. We compared the derived full model with predictions from the 3-variable 5-year kidney failure risk equation (KFRE) which includes age, sex and eGFR, and an elastic net model containing only these 3 variables [14]. We chose the 3-variable KFRE as albumin-to-creatine ratio was poorly recorded before lithium initiation and the 3variable model performed well in previous validation studies (ROC area 0.79) [22].

\section{External validation}

We used patient data from CPRD Gold for external validation of the model generated in the Aurum cohort. To categorise individuals at high risk of a rapid decline in eGFR, we ran group-based trajectory models of the 
eGFRs independently of the Aurum patients' trajectory model. We compared trajectory group membership with the predicted group membership from the Aurum model. We reported the ROC area, sensitivity and specificity at the cut-off point defined in the development data, brier score, predictive accuracy, calibration belt (a graphical approach designed to evaluate the goodness of fit of binary outcome models) [23] and decision curve analysis. We examined how well the model could predict CKD stage $3 \mathrm{~b}$ or more severe (eGFR $<45 \mathrm{~mL} / \mathrm{min} /$ $1.73 \mathrm{~m}^{2}$ ) during follow-up. We also compared the full model with simple models: the 3-variable KFRE and 3variable elastic net.

\section{Post hoc supplementary analysis}

We combined data from the Aurum and Gold datasets for patients who initiated lithium treatment with a baseline eGFR $\geq 90 \mathrm{~mL} / \mathrm{min} / 1.73 \mathrm{~m}^{2}$. We adopted the same approach in this smaller cohort: we identified a high-risk trajectory group using group-based trajectory modelling and then used the full model, 3-variable KFRE and 3variable elastic net to predict group membership. We also examined how well this model could predict CKD stage $3 \mathrm{a}$ or more severe (eGFR $<60 \mathrm{~mL} / \mathrm{min} /$ $1.73 \mathrm{~m}^{2}$ ) during follow-up. This analysis was completed to address issues arising from the strong association between baseline eGFR and future eGFR measurements in the initial model. All analysis was completed using Stata 16 [24].

\section{Results}

We identified 1609 patients in the development sample (Aurum cohort), with a median of 14 (IQR 7-26) eGFR test results each. The median length of lithium treatment was 1.42 years (IQR $0.53-3.58$ ). Of these patients, 401 (24.92\%) developed CKD stage 3a or more severe $\left(\right.$ eGFR $\left.<60 \mathrm{~mL} / \mathrm{min} / 1.73 \mathrm{~m}^{2}\right), 38$ (2.36\%) CKD stage $3 \mathrm{~b}\left(\mathrm{eGFR}<45 \mathrm{~mL} / \mathrm{min} / 1.73 \mathrm{~m}^{2}\right)$, but none developed CKD stage 4. In total, 158 (9.82\%) died during follow-up.

To categorise risk groups based on eGFR trajectories we chose a 5-group model, all groups with cubic trajectories (BIC $=3566.99)$. This defined $11.87 \%$ of the cohort as high risk. Models with 6 groups had lower BIC but included one group with less than $10 \%$ of the cohort.

Trajectories of the high-risk vs other groups (combined group 2-5) are shown in Fig. 1 and described in Table 1. Of those in the high-risk group 168 (87.96\%) develop CKD stage $3 \mathrm{a}$ or more severe, and 25 (13.09\%) developed stage $3 \mathrm{~b}$ or more severe, compared to $233(16.43 \%)$ and $13(0.92 \%)$ respectively in the low-risk group.

Patients in the high-risk group were more likely to be female, younger at lithium initiation, have a lower eGFR before starting lithium and be obese. They were more likely to have a pre-existing diagnosis of migraine. They were more likely to have a record of high LDL cholesterol. They were less likely to have had an eGFR $<60$ $\mathrm{mL} / \mathrm{min} / 1.73 \mathrm{~m}^{2}$ any time before baseline.

There was no statistical evidence of a difference in duration of lithium treatment and incidence of lithium toxicity ( $>1.5 \mathrm{mmol} / \mathrm{L}$ ) between groups. Those in the high-risk group were less likely to die during follow-up and had fewer eGFR tests in total.

We used 44 features known to the clinician prior to lithium initiation to generate a prediction model for being in the high-risk group. Elastic net with 10 -fold crossvalidation fitted a model with $\lambda=0.014$ and $\alpha=1.00$.

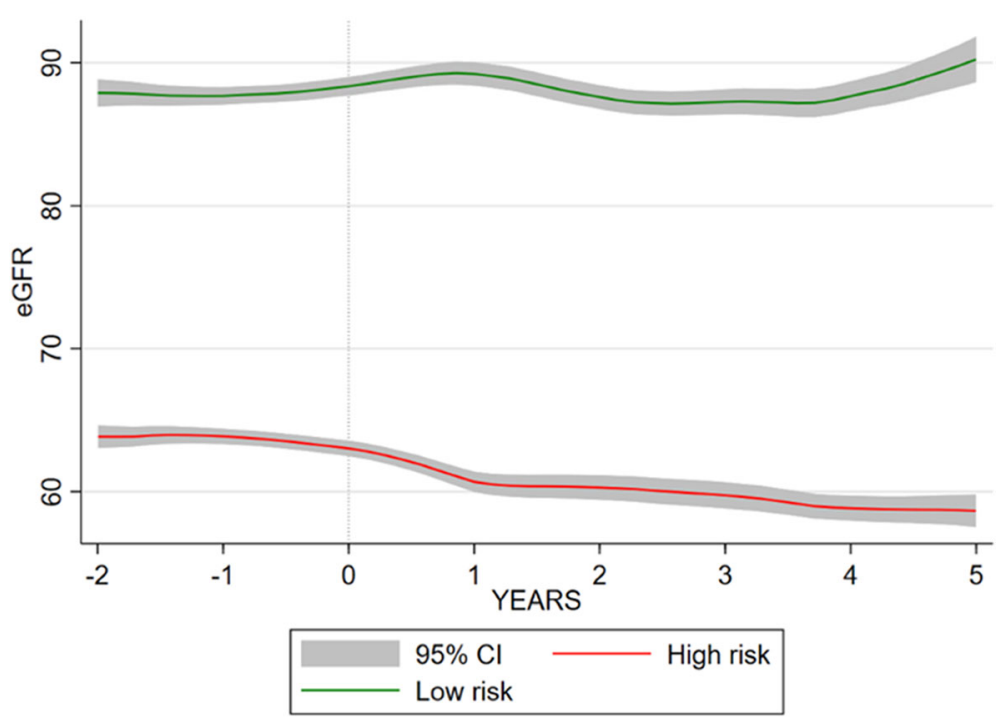

Fig. 1 High-risk and low-risk eGFR trajectory in relation to end of lithium exposure in Aurum 
Table 1 Characteristics of lithium prescribed patients by risk group in Aurum

\begin{tabular}{|c|c|c|c|}
\hline Feature & High-risk & Low-risk & $P$ value \\
\hline \multicolumn{4}{|l|}{ Patient characteristics } \\
\hline Total, $N(\%)$ & $191(11.87)$ & $1418(88.13)$ & \\
\hline Female, $n(\%)$ & $178(93.19)$ & $852(60.08)$ & $<0.001$ \\
\hline Age, median (IQR) & $41.58(32.14-50.72)$ & $45.80(36.39-56.59)$ & $<0.001$ \\
\hline BAME, $n(\%)$ & $7(3.66)$ & $78(5.50)$ & 0.287 \\
\hline In a relationship, $n(\%)$ & $32(16.75)$ & $240(16.93)$ & 0.953 \\
\hline Death during follow-up & $5(2.62)$ & $153(10.79)$ & $<0.001$ \\
\hline \multicolumn{4}{|l|}{ Lithium exposure characteristics } \\
\hline Lithium treatment duration (years), median (IQR) & $1.66(0.72-4.11)$ & $1.51(0.59-3.85)$ & 0.482 \\
\hline Ever lithium toxic (> $1.5 \mathrm{mmol} / \mathrm{L}), n(\%)$ & $5(2.62)$ & $62(4.37)$ & 0.256 \\
\hline Follow-up after stopping lithium (years), median (IQR) & $2.92(0-7.14)$ & $4.07(0.24-9.43)$ & 0.064 \\
\hline \multicolumn{4}{|l|}{ Kidney function characteristics } \\
\hline eGFR tests during follow-up, median (IQR) & $12(7-20)$ & $14(8-27)$ & 0.004 \\
\hline Baseline eGFR (mL/min/1.73 m²), median (IQR) & $67(63-72)$ & $81(72-91)$ & $<0.001$ \\
\hline Developed CKD stage 3a or more severe (eGFR $<60 \mathrm{~mL} / \mathrm{min} / 1.73 \mathrm{~m}^{2}$ ) & $168(87.96)$ & $233(16.43)$ & $<0.001$ \\
\hline Developed CKD stage 3b or more severe (eGFR $<45 \mathrm{~mL} / \mathrm{min} / 1.73 \mathrm{~m}^{2}$ ) & $25(13.09)$ & $13(0.92)$ & $<0.001$ \\
\hline \multicolumn{4}{|l|}{ Pre-lithium mental health characteristics } \\
\hline Depression, $n(\%)$ & $160(83.77)$ & $1167(82.30)$ & 0.616 \\
\hline Anxiety, $n(\%)$ & $60(31.41)$ & $403(28.42)$ & 0.391 \\
\hline Psychosis, $n$ (\%) & $33(17.28)$ & $266(18.76)$ & 0.621 \\
\hline Stress, $n(\%)$ & $45(23.56)$ & $262(18.48)$ & 0.093 \\
\hline Self-harm, $n(\%)$ & $39(20.42)$ & $217(15.30)$ & 0.070 \\
\hline Disturbed sleep, $n(\%)$ & $48(25.13)$ & $315(22.21)$ & 0.365 \\
\hline Illness duration (years), median (IQR) & $8.47(4.03-15.65)$ & $8.45(2.86-16.30)$ & 0.931 \\
\hline \multicolumn{4}{|l|}{ Pre-lithium physical health characteristics } \\
\hline Hypertension, n (\%) & $33(17.28)$ & $260(18.34)$ & 0.722 \\
\hline Migraine, $n(\%)$ & $40(20.94)$ & $138(9.73)$ & $<0.001$ \\
\hline Type II diabetes mellitus, $n$ (\%) & $18(9.42)$ & $107(7.55)$ & 0.363 \\
\hline \multicolumn{4}{|l|}{ Thyroid disease } \\
\hline Hypothyroidism, n (\%) & $5(2.62)$ & $70(4.94)$ & 0.154 \\
\hline Hyperthyroidism, n (\%) & $(-)^{*}$ & $8(0.56)$ & 0.113 \\
\hline \multicolumn{4}{|l|}{ Calcium abnormalities } \\
\hline hypocalcaemia, $n$ (\%) & $(-)^{*}$ & $12(0.85)$ & 0.640 \\
\hline hypercalcaemia, $n(\%)$ & $(-)^{*}$ & $13(0.93)$ & 0.184 \\
\hline \multicolumn{4}{|l|}{ Cholesterol abnormalities } \\
\hline High LDL, $n(\%)$ & $47(24.61)$ & $264(18.62)$ & 0.049 \\
\hline Low HDL, $n(\%)$ & $16(8.38)$ & $101(7.12)$ & 0.531 \\
\hline Asthma, $n(\%)$ & $52(27.23)$ & $282(19.89)$ & 0.019 \\
\hline Chronic obstructive pulmonary disease, $n(\%)$ & $57(29.84)$ & $341(24.05)$ & 0.081 \\
\hline Anaemia, $n(\%)$ & $11(5.76)$ & $67(4.72)$ & 0.532 \\
\hline Peptic ulcer, $n(\%)$ & $2(1.05)$ & $20(1.41)$ & 0.685 \\
\hline Coronary heart disease, $n(\%)$ & $5(2.62)$ & $68(4.80)$ & 0.175 \\
\hline Liver disease, $n(\%)$ & $(-)^{*}$ & $23(1.62)$ & 0.546 \\
\hline Neurological disorders, $n$ (\%) & $15(7.85)$ & $89(6.28)$ & 0.405 \\
\hline
\end{tabular}


Table 1 Characteristics of lithium prescribed patients by risk group in Aurum (Continued)

\begin{tabular}{|c|c|c|c|}
\hline Feature & High-risk & Low-risk & $P$ value \\
\hline Rheumatoid arthritis, $n(\%)$ & $6(3.14)$ & $28(1.97)$ & 0.293 \\
\hline Weight loss, n (\%) & $8(4.19)$ & $31(2.19)$ & 0.091 \\
\hline Ever eGFR $<60 \mathrm{~mL} / \mathrm{min} / 1.73 \mathrm{~m}^{2}, n(\%)$ & $(\downarrow)^{*}$ & $40(2.82)$ & 0.019 \\
\hline \multicolumn{4}{|l|}{ Health behaviours } \\
\hline Smoking status, $n(\%)$ & & & 0.51 \\
\hline Never smoked & $56(29.32)$ & $403(28.42)$ & \\
\hline Current smoker & $93(48.69)$ & $581(41.47)$ & \\
\hline Ex-smoker & $42(21.99)$ & $427(30.11)$ & \\
\hline Body mass index, $n(\%)$ & & & $<0.001$ \\
\hline Underweight & $(-)^{*}$ & $37(2.61)$ & \\
\hline Healthy weight & $57(29.84)$ & $616(43.44)$ & \\
\hline Overweight & $50(26.18)$ & 409 (28.84) & \\
\hline Obese & $80(41.88)$ & $356(25.11)$ & \\
\hline Cannabis use, $n(\%)$ & $(-)^{*}$ & $14(0.99)$ & 0.938 \\
\hline Other substance misuse, $n(\%)$ & $10(5.24)$ & 99 (6.98) & 0.367 \\
\hline Alcohol misuse, $n(\%)$ & $10(5.24)$ & $82(5.78)$ & 0.760 \\
\hline \multicolumn{4}{|l|}{ Other psychiatric drug treatments } \\
\hline Antipsychotic previously, $n$ (\%) & $104(54.45)$ & $766(54.02)$ & 0.911 \\
\hline Mood stabiliser previously, n (\%) & $72(37.70)$ & $432(30.47)$ & 0.043 \\
\hline SSRI previously, $n(\%)$ & $50(26.18)$ & 419 (29.55) & 0.336 \\
\hline TCA previously, $n(\%)$ & $55(28.80)$ & $334(23.55)$ & 0.112 \\
\hline Other antidepressant previously, $n$ (\%) & $43(22.51)$ & $236(16.64)$ & 0.044 \\
\hline
\end{tabular}

${ }^{{ }^{n}}<5$ individuals, $(\downarrow)$ lower \% in group, $(\uparrow)$ higher \% in group, (-) no evidence of difference between groups

The ROC area $=0.868$ (95\%CI 0.844-0.891) (Fig. 2). The empirical optimal cut-point was 0.134 with a sensitivity of $0.86(95 \% \mathrm{CI} \quad 0.78-0.94)$ and a specificity of 0.73 (95\%CI 0.63-0.84). The Youden index was 0.589. This gave a prediction accuracy of $74.54 \%$ (Table 2).

Features retained in the model were (in order of coefficient size): baseline eGFR, sex, sex by BMI group interaction, baseline eGFR by age interaction, hypothyroidism, migraine, BMI group, SSRI exposure, high LDL cholesterol, BAME, hyperthyroidism, smoking status, type 2 diabetes mellitus, and self-harm. The 3variable KFRE and the 3-variable elastic net model performed similarly well to the full model: ROC area $=$ 0.828 (95\%CI 0.801-0.855) and ROC area $=0.852$ (95\%CI 0.827-0.876), respectively (Table 2).

\section{External validation}

The external validation data set (Gold cohort) included 934 individuals. We developed new trajectory groups independently for these patients. BIC in the group-based trajectory model was minimised by a 5-group solution, with cubic or quadratic polynomials fitted for each group trajectory; 3, 2, 2, 3, 3 respectively from "highest risk" to "lowest risk" groups $(\mathrm{BIC}=1919.07)$. Of the total Gold cohort, $14.67 \%$ ( $n=137$ ) were in the high-risk group. Of the total cohort, 229 (24.52\%) developed CKD stage 3a or more severe and 14 (1.50\%) CKD stage $3 \mathrm{~b}$ or more severe.

Patient characteristics by risk group are described in Table 3, and trajectories relative to end of lithium exposure are shown in Fig. 3. Of those in the high-risk group, $117(85.40 \%)$ develop CKD stage 3a or more severe, and $14(10.22 \%)$ developed stage $3 \mathrm{~b}$ or more severe, compared to $112(14.05 \%)$ and < 5 respectively in the low-risk group.

As with the Aurum cohort, patients in the high-risk group were more likely to be female, be younger, have a lower eGFR before starting lithium and less likely to have a prior record of eGFR $<60 \mathrm{~mL} / \mathrm{min} / 1.73 \mathrm{~m}^{2}$. Highrisk individuals were also more likely to experience migraine. Unlike the Aurum cohort, the high-risk group were more likely to have anaemia and less likely to have hypertension. There was no between group difference for lithium duration and lithium toxicity was potentially more common in the low-risk group.

We predicted high-risk group membership using the model generated in the Aurum Data set. The ROC area was 0.879 (95\%CI 0.853-0.904) (Table 2, Fig. 4). At the empirical optimal cut-point defined in the development dataset, the model had a sensitivity of $0.91(0.84-0.97)$ and 


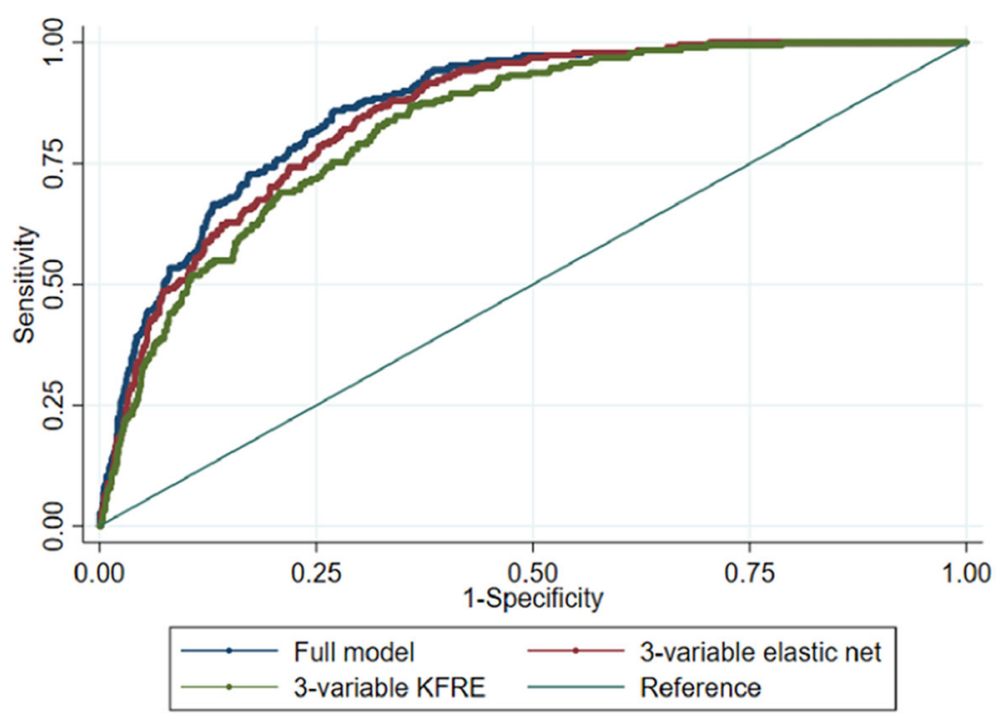

Fig. 2 Sensitivity vs specificity of the high-risk trajectory prediction model in Aurum

a specificity of 0.74 (95\% CI $0.67-0.82)$ The Brier score was 0.0967 . This gave a predictive accuracy of $76.55 \%$. However, the simpler models also predicted high-risk group membership similarly well: 3 -variable KFRE ROC area $=0.870(95 \%$ CI $0.841-0.898)$, 3-variable elastic net 0.888 (95\%CI 0.864-0.912) (Eq. 1). The calibration plot suggested that the model performs well up to a probability of 0.60 at the $95 \%$ confidence level, the calibration slope was 1.29 and calibration-in the-large 0.41 (Fig. 5).

Equation 1. 3-variable equation for prediction of high-risk group membership

Risk score $=4.621-(0.717 \times$ eGFR $)-\left(0.616 x_{\text {sex }}^{\dagger}\right)-(0.008 \times$ age $)$

probability of high-risk group membership $=\exp ($ risk score $) /(1+\exp ($ risk score $))$

${ }^{t}$ where $\operatorname{sex}=1$ if male and sex $=0$ if female

We also predicted CKD 3b or more severe using these models: $\mathrm{ROC}$ area $=0.849(95 \% \mathrm{CI} 0.792-0.905)$, $\mathrm{ROC}$ area $=0.865 \quad(95 \% \mathrm{CI} \quad 0.808-0.922), \quad$ ROC area $=0.858$ (95\%CI 0.792-0.922) using the full model, 3-variable elastic net and 3-variable KFRE respectively (Table 4).
The decision curve analysis showed that all 3 of these models were superior to classifying everyone as high risk or low risk between a threshold probability of 0.10 and 0.80 and there was little difference between them (Fig. 6).

\section{Post hoc supplementary analysis}

In 668 patients with a baseline eGFR $\geq 90 \mathrm{~mL} / \mathrm{min} /$ $1.73 \mathrm{~m}^{2}$ a two-group cubic trajectory model minimised the BIC (642.39) with 120 patients $(17.96 \%)$ in the highrisk group (Table 5, Fig. 7). CKD stage 3a or more severe and stage $3 \mathrm{~b}$ or more severe were more common in the high-risk group. Individuals in the high-risk group were again more likely to be female, be younger and have a lower eGFR before starting lithium. They were more likely to be current smokers. We did not observe any of the other between group differences present in the Aurum or Gold trajectory groups.

In this reduced dataset, our full model was better at predicting high-risk group membership than the 3-

Table 2 Prediction of high-risk group membership

\begin{tabular}{|c|c|c|c|c|}
\hline Model & ROC area $(95 \% \mathrm{Cl})$ & Sensitivity $(95 \% \mathrm{Cl})$ & Specificity $(95 \% \mathrm{Cl})$ & Accuracy $\%$ \\
\hline \multicolumn{5}{|l|}{ Development (Aurum) } \\
\hline Full & $0.868(95 \% \mathrm{Cl} 0.844-0.891)$ & $0.86(0.78-0.94)$ & $0.73(0.66-0.80)$ & 74.54 \\
\hline 3-variable KFRE & $0.828(0.801-0.855)$ & $0.87(0.77-0.96)$ & $0.64(0.54-0.74)$ & 66.73 \\
\hline 3-variable elastic net & $0.852(0.827-0.856)$ & $0.86(0.77-0.95)$ & $0.68(0.58-0.78)$ & 70.14 \\
\hline \multicolumn{5}{|l|}{ Validation (Gold) } \\
\hline Full & $0.879(0.853-0.904)$ & $0.91(0.84-0.97)$ & $0.74(0.67-0.81)$ & 76.55 \\
\hline 3-variable KFRE & $0.870(0.841-0.898)$ & $0.86(0.78-0.94)$ & $0.75(0.68-0.83)$ & 76.66 \\
\hline 3-variable elastic net & $0.888(0.864-0.912)$ & $0.86(0.79-0.94)$ & $0.80(0.73-0.87)$ & 80.90 \\
\hline
\end{tabular}


Table 3 Characteristics of lithium prescribed patients by risk group in Gold

\begin{tabular}{|c|c|c|c|}
\hline Feature & High-risk & Low-risk & $P$ value \\
\hline \multicolumn{4}{|l|}{ Patient characteristics } \\
\hline Total, $N(\%)$ & $137(14.67)$ & $797(85.33)$ & \\
\hline Female, $n(\%)$ & $129(94.16)$ & $466(58.47)$ & $<0.001$ \\
\hline Age, median (IQR) & $41.07(32.68-49.95)$ & 46. 75 (36.84-59.27) & 0.001 \\
\hline BAME, $n(\%)$ & $2(1.46)$ & $10(1.25)$ & 0.844 \\
\hline In a relationship, $n(\%)$ & $34(24.82)$ & $150(18.82)$ & 0.103 \\
\hline Death during follow-up & $9(6.57)$ & $80(10.04)$ & 0.202 \\
\hline \multicolumn{4}{|l|}{ Lithium exposure characteristics } \\
\hline Lithium treatment duration (years), median (IQR) & $1.23(0.37-2.66)$ & $1.23(0.44-3.10)$ & 0.926 \\
\hline Ever lithium toxic (> $1.5 \mathrm{mmol} / \mathrm{L}), n(\%)$ & $(\downarrow)^{*}$ & $37(1.68)$ & 0.048 \\
\hline Follow-up after stopping lithium (years), median (IQR) & $3.43(0.42-7.36)$ & $4.44(0.81-8.31)$ & 0.165 \\
\hline \multicolumn{4}{|l|}{ Kidney function characteristics } \\
\hline eGFR tests during follow-up, median (IQR) & $11[6-21]$ & $14(7-24)$ & 0.031 \\
\hline Baseline eGFR (mL/min/1.73 m²), median (IQR) & $66(63-71)$ & $83(74-94)$ & $<0.001$ \\
\hline Developed CKD stage 3a or more severe (eGFR $<60 \mathrm{~mL} / \mathrm{min} / 1.73 \mathrm{~m}^{2}$ ) & $117(85.40)$ & $112(14.05)$ & $<0.001$ \\
\hline Developed CKD stage 3b or more severe (eGFR $<45 \mathrm{~mL} / \mathrm{min} / 1.73 \mathrm{~m}^{2}$ ) & $14(10.22)$ & $(\downarrow)^{*}$ & $<0.001$ \\
\hline \multicolumn{4}{|l|}{ Pre-lithium mental health characteristics } \\
\hline Depression, $n(\%)$ & $118(86.13)$ & $634(79.55)$ & 0.072 \\
\hline Anxiety, $n(\%)$ & $396(49.69)$ & $74(54.01)$ & 0.349 \\
\hline Psychosis, $n$ (\%) & $37(27.01)$ & $179(22.46)$ & 0.244 \\
\hline Stress, $n(\%)$ & $25(18.25)$ & $138(17.31)$ & 0.790 \\
\hline Self-harm, $n(\%)$ & $40(29.20)$ & $185(23.21)$ & 0.130 \\
\hline Disturbed sleep, $n(\%)$ & $49(35.77)$ & $202(25.35)$ & 0.011 \\
\hline Illness duration (years), median (IQR) & $7.73(2.55-13.80)$ & $7.65(2.71-15.48)$ & 0.926 \\
\hline \multicolumn{4}{|l|}{ Pre-lithium physical health characteristics } \\
\hline Hypertension, n (\%) & 15 (10.95) & $165(20.70)$ & 0.008 \\
\hline Migraine, $n(\%)$ & $24(17.52)$ & $79(9.91)$ & 0.009 \\
\hline Type II diabetes mellitus, $n$ (\%) & $10(7.30)$ & $64(8.03)$ & 0.770 \\
\hline \multicolumn{4}{|l|}{ Thyroid disease } \\
\hline Hypothyroidism, n (\%) & $15(10.95)$ & $57(7.15)$ & 0.124 \\
\hline Hyperthyroidism, n (\%) & $(-)^{*}$ & $5(0.63)$ & 0.353 \\
\hline \multicolumn{4}{|l|}{ Calcium abnormalities } \\
\hline Hypocalcaemia, $n$ (\%) & $(-)^{*}$ & $9(1.13)$ & 0.740 \\
\hline Hypercalcaemia, $n(\%)$ & $(-)^{*}$ & $(-)^{*}$ & 0.678 \\
\hline \multicolumn{4}{|l|}{ Cholesterol abnormalities } \\
\hline High LDL, $n(\%)$ & $20(14.60)$ & $164(20.58)$ & 0.104 \\
\hline Low HDL, $n(\%)$ & $10(7.30)$ & $66(8.28)$ & 0.698 \\
\hline Asthma, $n(\%)$ & $30(21.90)$ & $131(16.44)$ & 0.118 \\
\hline Chronic obstructive pulmonary disease, $n(\%)$ & $36(26.28)$ & $162(20.33)$ & 0.115 \\
\hline Anaemia, $n(\%)$ & $12(8.76)$ & $30(3.76)$ & 0.009 \\
\hline Peptic ulcer, $n(\%)$ & $6(4.38)$ & $27(3.39)$ & 0.561 \\
\hline Coronary heart disease, $n(\%)$ & $(\downarrow)^{*}$ & $46(5.77)$ & 0.035 \\
\hline Liver disease, $n(\%)$ & $(-)^{*}$ & $10(1.25)$ & 0.138 \\
\hline Neurological disorders, $n$ (\%) & $10(7.30)$ & $45(5.65)$ & 0.448 \\
\hline
\end{tabular}


Table 3 Characteristics of lithium prescribed patients by risk group in Gold (Continued)

\begin{tabular}{|c|c|c|c|}
\hline Feature & High-risk & Low-risk & $P$ value \\
\hline Rheumatoid arthritis, $n(\%)$ & $(-)^{*}$ & $17(2.13)$ & 0.606 \\
\hline Weight loss, n (\%) & $5(3.65)$ & $25(3.14)$ & 0.753 \\
\hline Ever eGFR< $60 \mathrm{~mL} / \mathrm{min} / 1.73 \mathrm{~m}^{2}, n(\%)$ & $(\downarrow)^{*}$ & $105(13.17)$ & $<0.001$ \\
\hline \multicolumn{4}{|l|}{ Health behaviours } \\
\hline Smoking status, $n(\%)$ & & & 0.048 \\
\hline Never smoked & $55(40.15)$ & $297(37.26)$ & \\
\hline Current smoker & $65(47.45)$ & $329(41.28)$ & \\
\hline Ex-smoker & $17(12.41)$ & $171(21.46)$ & \\
\hline Body mass index, $n(\%)$ & & & 0.259 \\
\hline Underweight & $(-)^{*}$ & $9(1.13)$ & \\
\hline Healthy weight & $58(42.34)$ & $321(40.28)$ & \\
\hline Overweight & $34(24.82)$ & $257(32.25)$ & \\
\hline Obese & $42(30.66)$ & $210(26.35)$ & \\
\hline Cannabis use, $n(\%)$ & $(-)^{*}$ & $13(1.63)$ & 0.297 \\
\hline Other substance misuse, $n(\%)$ & $(-)^{*}$ & $21(2.63)$ & 0.849 \\
\hline Alcohol misuse, $n(\%)$ & $29(21.17)$ & $111(13.93)$ & 0.028 \\
\hline \multicolumn{4}{|l|}{ Other drug treatment } \\
\hline Antipsychotic previously, $n$ (\%) & $73(53.28)$ & $432(54.20)$ & 0.842 \\
\hline Mood stabiliser previously, n (\%) & $38(27.74)$ & $156(19.57)$ & 0.030 \\
\hline SSRI previously, $n(\%)$ & $43(31.39)$ & $211(26.47)$ & 0.233 \\
\hline TCA previously, $n(\%)$ & $35(25.55)$ & $193(24.22)$ & 0.737 \\
\hline Other antidepressant previously, $n$ (\%) & $79(57.66)$ & $391(49.06)$ & 0.063 \\
\hline
\end{tabular}

${ }^{{ }^{*} n<5}$ individuals, $(\downarrow)$ lower \% in group, $(\uparrow)$ higher \% in group, $(-)$ no evidence of difference between groups

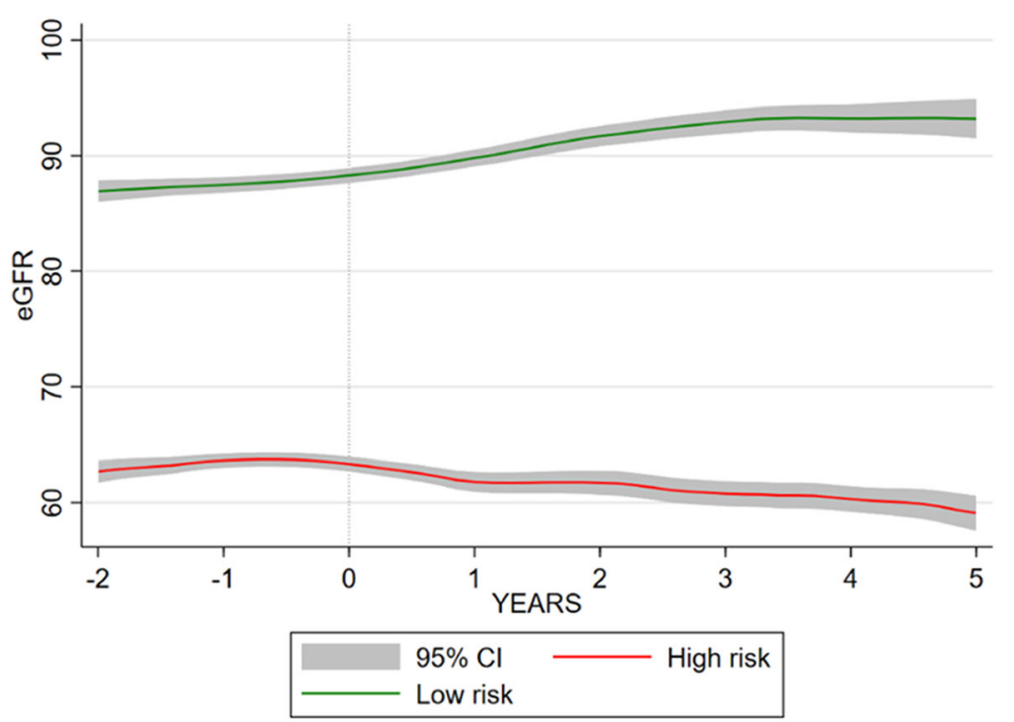

Fig. 3 High-risk and low-risk eGFR trajectory in relation to stopping lithium in Gold 


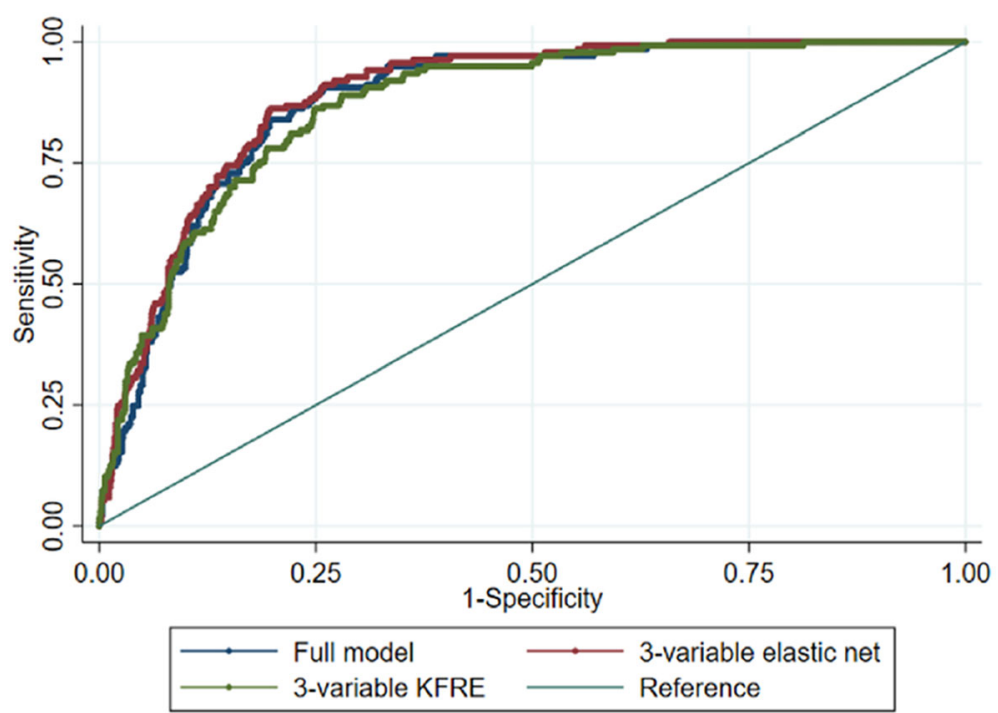

Fig. 4 Sensitivity vs specificity of the high-risk trajectory prediction model in Gold

variable KFRE model $(\mathrm{ROC}$ area $=0.725$; $95 \% \mathrm{CI} 0.675-$ 0.776 vs $\mathrm{ROC}$ area $=0.667 ; 95 \%$ CI $0.617-0.716 ; p$ value for equality $=0.0018$ ), but not the 3 -variable elastic net model $($ ROC area $=0.729 ; 95 \%$ CI $0.679-0.779 ; p$ value for equality 0.5846) (Table 6, Fig. 8).

\section{Discussion}

As far as we are aware, this is the first model developed to predict high risk of future eGFR decline in people with bipolar disorder prescribed lithium. We used a large representative sample of people with bipolar disorder initiated on lithium and followed up for up for a median of 7.10 years (IQR 3.85-11.36). It is also the first study to use the two CPRD datasets, covering a large, representative sample of the UK population to develop a prediction model and provide external validation.

Because of the rarity of kidney failure, and the varied follow-up time and eGFR recording frequency in EHRs, we sought to identify approximately $10 \%$ of individuals prescribed lithium who were at highest risk of deteriorating kidney function, defined by the trajectory of their serial eGFR measurements. Using group-based trajectory analysis we identified high-risk groups independently in the Aurum and Gold cohorts. In both cases approximately $85 \%$ of those categorised as high risk developed CKD stage $3 \mathrm{a}$ or more severe compared to

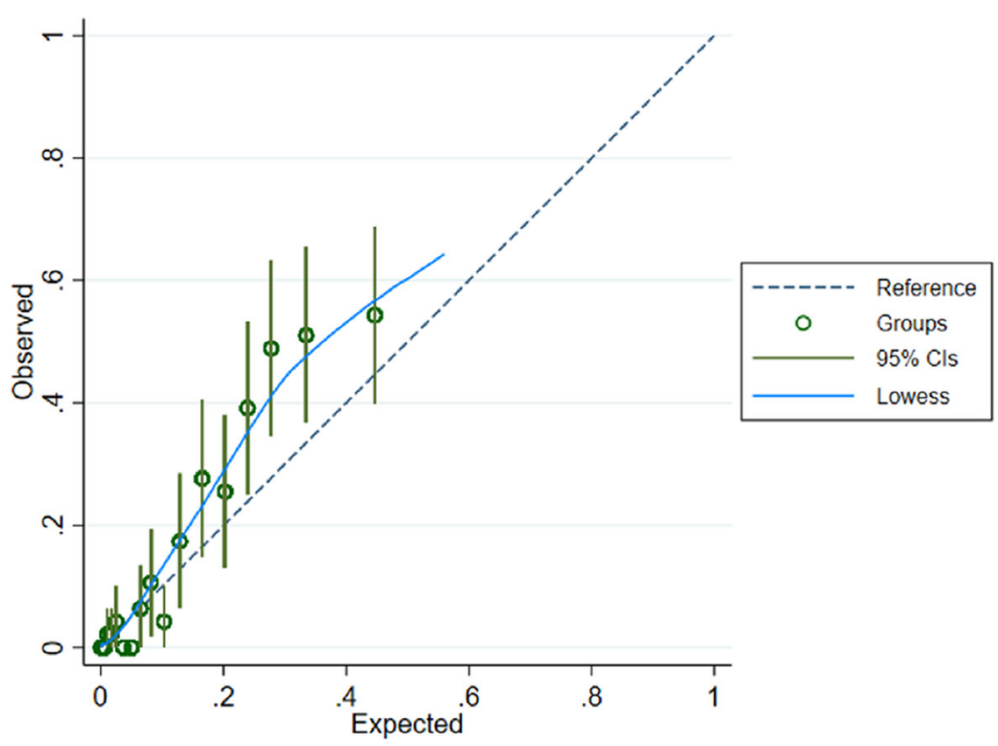

Fig. 5 Calibration plot for Gold data set (20 groups across risk spectrum) 
Table 4 Prediction of CKD stage 3b or more severe

\begin{tabular}{|c|c|c|c|c|}
\hline MODEL & ROC area $(95 \% \mathrm{Cl})$ & Sensitivity $(95 \% \mathrm{Cl})$ & Specificity $(95 \% \mathrm{Cl})$ & Accuracy, \% \\
\hline \multicolumn{5}{|l|}{ Development (Aurum) } \\
\hline Full & $0.700(0.617-0.783)$ & $0.71(0.52-0.90)$ & $0.66(0.47-0.85)$ & 66.12 \\
\hline 3-variable KFRE & $0.627(0.543-0.710)$ & $0.68(0.51-0.86)$ & $0.61(0.48-0.74)$ & 61.18 \\
\hline 3-variable elastic net & $0.678(0.598-0.758)$ & $0.58(0.37-0.78)$ & $0.73(0.48-0.98)$ & 72.64 \\
\hline \multicolumn{5}{|l|}{ Validation (Gold) } \\
\hline Full & $0.849(0.792-0.905)$ & $1.00(0.96-1.00)$ & $0.67(0.57-0.76)$ & 67.49 \\
\hline 3-variable KFRE & $0.858(0.792-0.922)$ & $0.86(0.70-1.00)$ & $0.78(0.63-0.93)$ & 78.12 \\
\hline 3-variable elastic net & $0.865(0.808-0.921)$ & $1.00(0.92-1.00)$ & $0.66(0.57-0.75)$ & 66.51 \\
\hline
\end{tabular}

approximately $15 \%$ in the low-risk groups. Approximately $10 \%$ of those identified as high risk developed CKD stage $3 \mathrm{~b}$ or more severe, compared to $<1 \%$ in the low-risk group. A number of features differed between the high-risk and low-risk groups in both cohorts. Those in the high-risk groups were more likely to be female, younger, more likely to have a lower eGFR before starting lithium, more likely to experience migraine and less likely to have a prior record of eGFR $<60 \mathrm{~mL} / \mathrm{min} /$ $1.73 \mathrm{~m}^{2}$. These CKD risk factors have been previously identified. CKD is more common in women [25], and this has been shown in lithium users specifically [26]. Younger women appear to be at particular risk [26]. Low baseline eGFR increases risk of CKD in the general population [27]. Migraine is not commonly thought of as a risk factor for CKD, but has been identified as such in one study, especially in younger age groups [28]. Migraine is highly comorbid with bipolar disorder [29] and it may also be a proxy for medication use which could impair kidney function. In both cohorts, there was no association between duration of lithium treatment or lithium toxicity (which was rare) and high-risk group membership.

Our model, developed in CPRD Aurum to predict whether individuals were in a high-risk group for eGFR decline during treatment with lithium for bipolar disorder, had excellent discrimination in the CPRD Gold cohort $($ ROC area $=0.879)$. However, simple models only including sex, age and baseline eGFR performed similarly well (3-variable KFRE ROC area $=0.870$, 3-variable elastic net $\mathrm{ROC}$ area $=0.888$ ), all with similar levels of accuracy (>75\%). In the external validation data set, our model designed to predict high-risk trajectory predicted CKD stage $3 \mathrm{~b}$ or more severe (eGFR $<45 \mathrm{~mL} / \mathrm{min} /$ $1.73 \mathrm{~m}^{2}$ ) with a ROC area $=0.849$. Again, simple models performed well, with the 3 -variable KFRE having the highest accuracy (78\%).

When we reduced our cohort to those starting lithium with an eGFR $\geq 90 \mathrm{~mL} / \mathrm{min} / 1.73 \mathrm{~m}^{2}$ our full model and 3 -variable elastic net model performed better than the
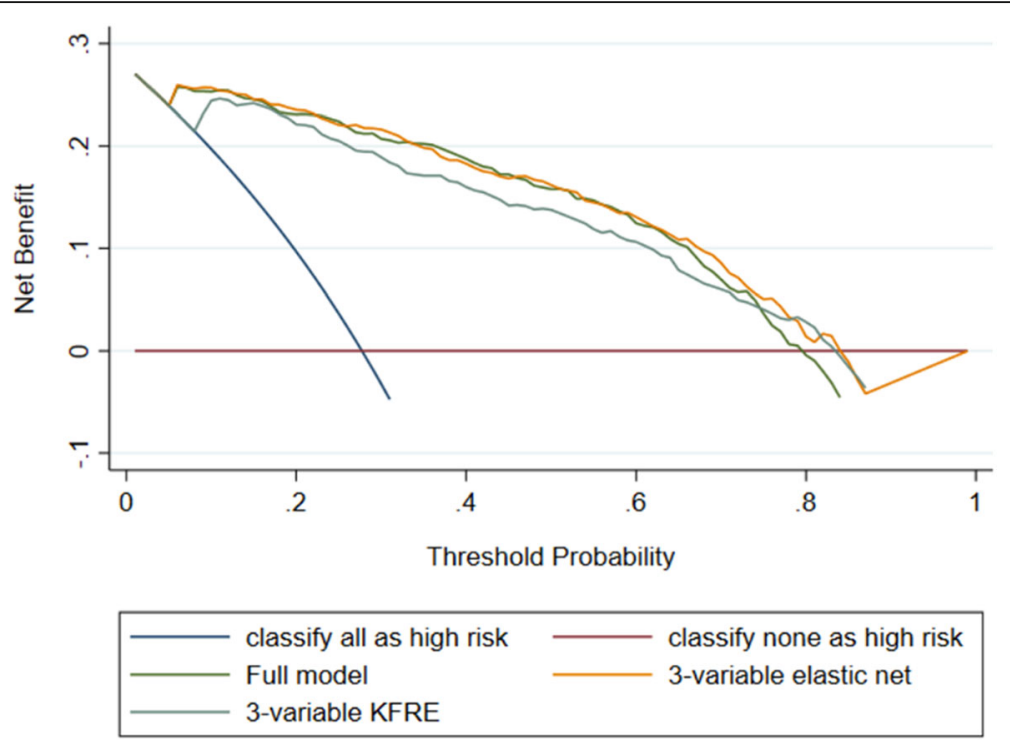

Fig. 6 Decision curve analysis for Gold data set 
Table 5 Characteristics of lithium prescribed patients by risk group in patients with baseline eGFR $\geq 90 \mathrm{~mL} / \mathrm{min} / 1.73 \mathrm{~m}{ }^{2}$

\begin{tabular}{|c|c|c|c|}
\hline Feature & High-risk & Low-risk & $P$ value \\
\hline \multicolumn{4}{|l|}{ Patient characteristics } \\
\hline Total, N (\%) & $120(17.96)$ & $548(82.04)$ & \\
\hline Female, $n(\%)$ & $65(54.17)$ & $158(28.83)$ & $<0.001$ \\
\hline Age, median (IQR) & $45.48(34.99-55.58)$ & $52.18(42.03-65.93)$ & $<0.001$ \\
\hline BAME, $n(\%)$ & $*(-)$ & $15(2.74)$ & 0.500 \\
\hline In a relationship, $n(\%)$ & $23(19.17)$ & $82(14.96)$ & 0.252 \\
\hline Death during follow-up & $13(10.83)$ & $94(17.15)$ & 0.087 \\
\hline \multicolumn{4}{|l|}{ Lithium exposure characteristics } \\
\hline Lithium treatment duration (years), median (IQR) & $1.23(0.54-3.07)$ & $1.61(0.61-3.84)$ & 0.087 \\
\hline Ever lithium toxic (> $1.5 \mathrm{mmol} / \mathrm{L}), n(\%)$ & $7(5.83)$ & $36(6.57)$ & 0.867 \\
\hline Follow-up after stopping lithium (years), median (IQR) & $8.01(2.68-12.08)$ & $4.77(0.97-9.77)$ & 0.001 \\
\hline \multicolumn{4}{|l|}{ Kidney function characteristics } \\
\hline Baseline eGFR (mL/min/1.73 m2), median (IQR) & $95(92-100)$ & $100(94-108)$ & $<0.001$ \\
\hline Developed CKD stage 3a or more severe (eGFR< 60 mL/min/1.73 m²) & $34(28.33)$ & $(\downarrow)^{*}$ & $<0.001$ \\
\hline Developed CKD stage $3 \mathrm{~b}$ or more severe (eGFR $<45 \mathrm{~mL} / \mathrm{min} / 1.73 \mathrm{~m}^{2}$ ) & $(\uparrow)^{*}$ & $(\downarrow)^{*}$ & $<0.001$ \\
\hline \multicolumn{4}{|l|}{ Pre-lithium mental health characteristics } \\
\hline Depression, $n(\%)$ & $97(80.83)$ & $423(77.19)$ & 0.384 \\
\hline Anxiety, $n(\%)$ & $34(28.33)$ & $188(34.31)$ & 0.208 \\
\hline Psychosis, $n$ (\%) & $26(21.67)$ & $133(24.27)$ & 0.544 \\
\hline Stress, $n(\%)$ & $14(11.67)$ & $71(12.96)$ & 0.701 \\
\hline Self-harm, $n(\%)$ & $24(20.00)$ & $84(15.33)$ & 0.208 \\
\hline Disturbed sleep, $n(\%)$ & $24(20.00)$ & $113(20.62)$ & 0.879 \\
\hline IIlness duration (years), median (IQR) & $7.43(1.81-12.87)$ & $7.26(2.53-17.79)$ & 0.687 \\
\hline \multicolumn{4}{|l|}{ Pre-lithium physical health characteristics } \\
\hline Hypertension, $n$ (\%) & $20(16.67)$ & $140(25.55)$ & 0.039 \\
\hline Migraine, $n(\%)$ & $9(7.50)$ & $35(6.39)$ & 0.656 \\
\hline Type II diabetes mellitus, $n$ (\%) & $7(5.83)$ & $58(10.58)$ & 0.112 \\
\hline \multicolumn{4}{|l|}{ Thyroid disease } \\
\hline Hypothyroidism, n (\%) & $(-)^{*}$ & $24(4.38)$ & 0.604 \\
\hline Hyperthyroidism, $n$ (\%) & $(-)^{*}$ & $(-)^{*}$ & 0.507 \\
\hline \multicolumn{4}{|l|}{ Calcium abnormalities } \\
\hline hypocalcaemia, $n$ (\%) & $(-)^{*}$ & $(-)^{*}$ & 0.934 \\
\hline hypercalcaemia, $n(\%)$ & $(-)^{*}$ & $6(1.09)$ & 0.799 \\
\hline \multicolumn{4}{|l|}{ Cholesterol abnormalities } \\
\hline High LDL, $n(\%)$ & $29(24.17)$ & $97(17.70)$ & 0.101 \\
\hline Low HDL, $n$ (\%) & $12(10.00)$ & $46(8.39)$ & 0.572 \\
\hline Asthma, $n(\%)$ & $25(20.83)$ & $75(13.69)$ & 0.047 \\
\hline Chronic obstructive pulmonary disease, $n(\%)$ & $29(24.17)$ & $100(18.25)$ & 0.137 \\
\hline Anaemia, $n(\%)$ & $(-)^{*}$ & $20(3.65)$ & 0.532 \\
\hline Peptic ulcer, $n(\%)$ & $(-)^{*}$ & $16(2.92)$ & 0.189 \\
\hline Coronary heart disease, $n(\%)$ & $5(4.17)$ & 49 (8.94) & 0.082 \\
\hline Liver disease, $n(\%)$ & $(-)^{*}$ & $8(1.46)$ & 0.590 \\
\hline Neurological disorders, $n$ (\%) & $6(5.00)$ & $41(7.48)$ & 0.4336 \\
\hline Rheumatoid arthritis, $n$ (\%) & $5(4.17)$ & $11(2.01)$ & 0.161 \\
\hline
\end{tabular}


Table 5 Characteristics of lithium prescribed patients by risk group in patients with baseline eGFR $\geq 90 \mathrm{~mL} / \mathrm{min} / 1.73 \mathrm{~m}{ }^{2}$ (Continued)

\begin{tabular}{|c|c|c|c|}
\hline Feature & High-risk & Low-risk & $P$ value \\
\hline Weight loss, $n(\%)$ & $(-)^{*}$ & $11(2.01)$ & 0.381 \\
\hline Ever eGFR< $60 \mathrm{~mL} / \mathrm{min} / 1.73 \mathrm{~m}^{2}, n(\%)$ & $16(13.33)$ & $105(19.16)$ & 0.133 \\
\hline \multicolumn{4}{|l|}{ Health behaviours } \\
\hline Smoking status, $n(\%)$ & & & 0.001 \\
\hline Never smoked & $26(21.67)$ & $197(35.95)$ & \\
\hline Current smoker & $62(51.67)$ & $193(35.22)$ & \\
\hline Ex-smoker & $32(26.67)$ & $158(28.83)$ & \\
\hline Body mass index, $n(\%)$ & & & 0.534 \\
\hline Underweight & $(-)^{*}$ & $9(1.28)$ & \\
\hline Healthy weight & $47(39.17)$ & $227(41.42)$ & \\
\hline Overweight & $36(30.00)$ & $187(34.12)$ & \\
\hline Obese & $35(29.17)$ & $127(23.18)$ & \\
\hline Cannabis use, $n(\%)$ & $(-)^{*}$ & $6(1.09)$ & 0.799 \\
\hline Other substance misuse, $n(\%)$ & $7(5.83)$ & $19(3.47)$ & 0.225 \\
\hline Alcohol misuse, $n(\%)$ & $8(6.67)$ & $47(8.58)$ & 0.491 \\
\hline \multicolumn{4}{|l|}{ Other drug treatments } \\
\hline Antipsychotic previously, $n$ (\%) & $65(54.17)$ & $269(49.09)$ & 0.314 \\
\hline Mood stabiliser previously, n (\%) & $34(28.33)$ & $117(21.35)$ & 0.098 \\
\hline SSRI previously, $n(\%)$ & $31(25.83)$ & $126(22.99)$ & 0.506 \\
\hline TCA previously, $n(\%)$ & $19(15.83)$ & $120(21.90)$ & 0.138 \\
\hline Other antidepressant previously, $n$ (\%) & $16(13.33)$ & $60(10.95)$ & 0.456 \\
\hline
\end{tabular}

${ }^{*} n<5$ individuals, ( $\left.\downarrow\right)$ lower \% in group, ( $\left.\uparrow\right)$ higher \% in group, (-) no evidence of difference between groups

KFRE. However, our sample was too small to complete external validation of these new models.

Given these findings, simple risk calculators should be used in clinical practice at the decision to commence lithium and when eGFR is regularly measured. This could be the KFRE or our 3-varaible elastic net model (Eq. 1), which performs better than the 3-variable KFRE when eGFR $\geq 90 \mathrm{~mL} / \mathrm{min} / 1.73 \mathrm{~m}^{2}$. We did not find predictors of eGFR decline that were specific to lithiumtreated patients.

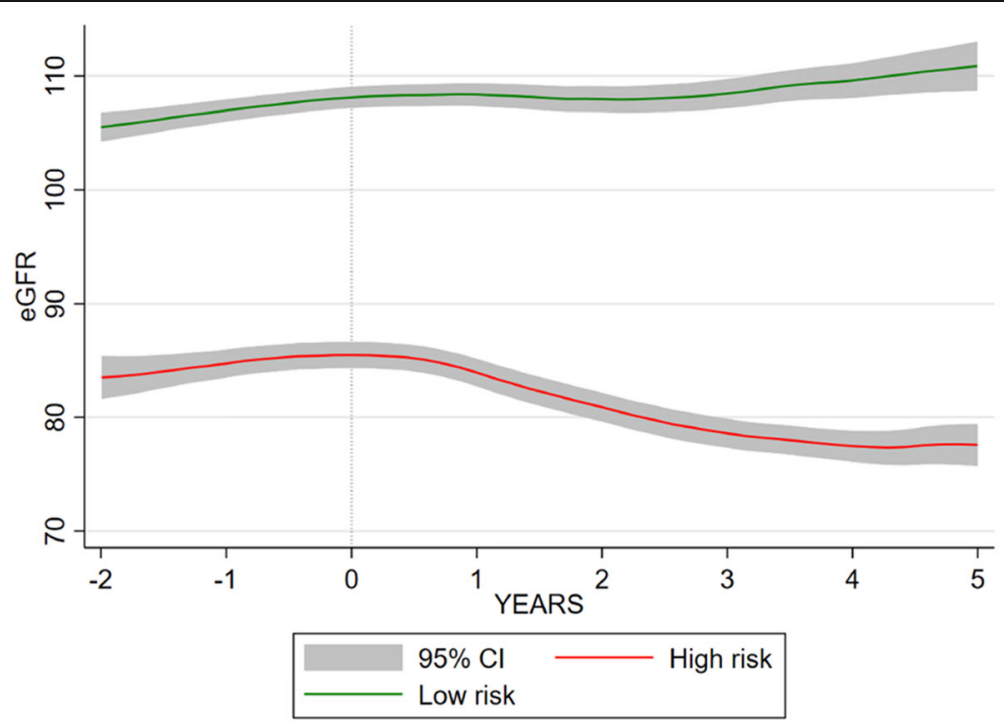

Fig. 7 High-risk and low-risk eGFR trajectory in relation to stopping lithium in patients with baseline eGFR $\geq 90 \mathrm{~mL} / \mathrm{min} / 1.73 \mathrm{~m}{ }^{2}$ 
Table 6 Prediction in individuals with baseline eGFR $\geq 90 \mathrm{~mL} / \mathrm{min} / 1.73 \mathrm{~m}^{2}$

\begin{tabular}{|c|c|c|c|c|}
\hline Model & ROC area $(95 \% \mathrm{Cl})$ & Sensitivity $(95 \% \mathrm{Cl})$ & Specificity $(95 \% \mathrm{Cl})$ & Accuracy, \% \\
\hline \multicolumn{5}{|l|}{ High-risk group } \\
\hline Full & $0.725(0.675-0.776)$ & $0.62(0.47-0.76)$ & $0.73(0.61-0.86)$ & 70.96 \\
\hline 3-variable KFRE & $0.667(0.617-0.716)$ & $0.70(0.48-0.92)$ & $0.57(0.32-0.81)$ & 59.33 \\
\hline 3-variable elastic net & $0.729(0.679-0.779)$ & $0.64(0.48-0.80)$ & $0.72(0.56-0.87)$ & 70.66 \\
\hline \multicolumn{5}{|l|}{ CKD 3a } \\
\hline Full & $0.757(0.667-0.846)$ & $0.59(0.38-0.81)$ & $0.80(0.63-0.97)$ & 78.89 \\
\hline 3-variable KFRE & $0.610(0.521-0.698)$ & $0.54(0.24-0.84)$ & $0.68(0.29-1.07)$ & 67.22 \\
\hline 3-variable elastic net & $0.748(0.657-0.839)$ & $0.56(0.64-0.75)$ & $0.83(0.64-1.00)$ & 81.59 \\
\hline
\end{tabular}

\section{Strengths and limitations}

Our large, population-based longitudinal sample avoided selection bias and is generalisable and representative. Our group-based trajectory approach avoided issues with differential follow-up time and potential surveillance bias. Our use of elastic net allowed us to build a parsimonious prediction model from a large number of potential features.

The study has a number of limitations. Instead of using precise eGFR values to define outcome, we split individuals into those with a high-risk and low-risk trajectory. We forced the group-based trajectory model to identify $>10 \%$ of individuals prescribed lithium who were at the highest risk of eGFR decline. A more useful model clinically would be to predict true kidney failure requiring intervention; however, this was too rare in our cohort ( $2 \%$ developed CKD stage $3 \mathrm{~b}$ or more severe), suggesting it is uncommon in modern clinical practice. We may also have been limited by the relatively short duration of lithium prescribing for many individuals included in the study. We included a large number of potential predictors. However, we may not have included all important features in our elastic net model. Some variables of interest, such as proteinuria, were poorly recorded and biased by diabetes diagnosis. We did not include the broad range of drugs for physical health problems that may influence eGFR, but we did include many physical health conditions for which these drugs would be indicated.

It is possible that we failed to identify people previously exposed to lithium, but we attempted to limit this by ensuring patients had at least a year of follow-up at the same primary care practice before their first identified lithium prescription. In most cases this would also include the uploading of historical records to the EHR. Patients could also be misclassified in terms of different features included in the model. However, our intention was to build a model based on what is already known about the patient from the EHR. Misclassification may be more likely for some features (for example, in a relationship) than others (for example, chronic obstructive pulmonary disease).

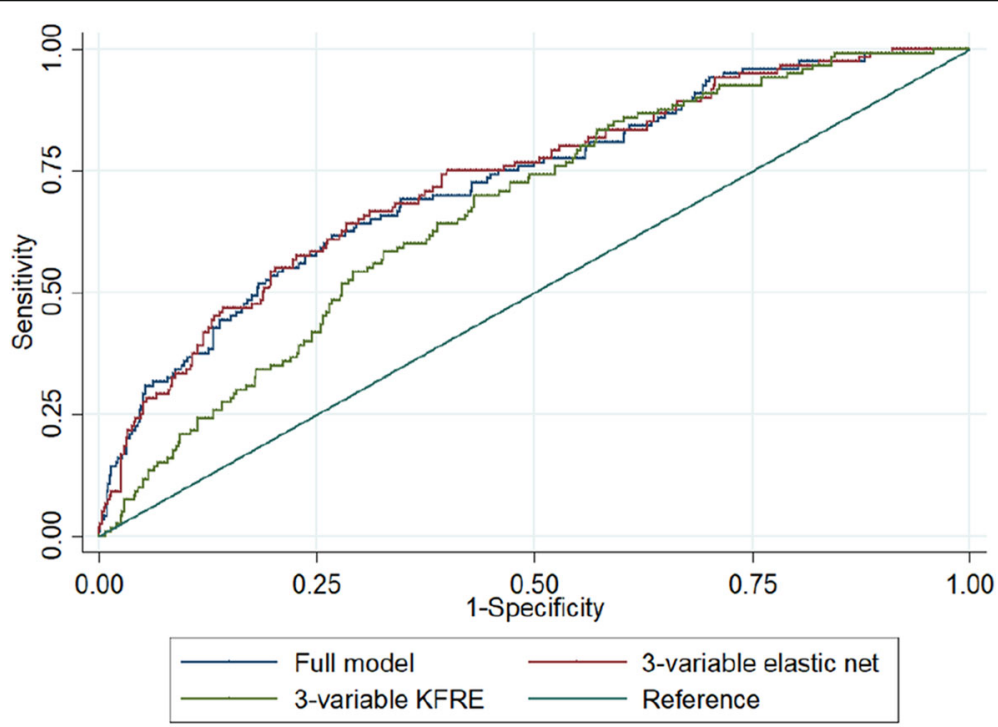

Fig. 8 Comparison of ROC areas between full model, 3-variable model and 3-variable KFRE model 
We initially planned to develop a model for individuals with essentially normal kidney function (eGFR $\geq 60 \mathrm{~mL} /$ $\min / 1.73 \mathrm{~m}^{2}$ ). Although the discrimination and calibration of the model was good, a simple model based on baseline eGFR, age and sex performed just as well.

\section{Conclusion}

We developed a model for predicting, at lithium initiation, individuals at high risk of a poor trajectory of kidney function using serial eGFR measurements. We externally validated this model, which had excellent discrimination and good calibration. CKD stage $3 \mathrm{~b}$ or more severe occurred in $2 \%$ of the population across the two cohorts. Whilst this is worrying, it means that the vast majority of patients treated with lithium do not develop kidney failure, and those at risk can be identified prior to initiating lithium using their age, sex and baseline eGFR.

\section{Authors' contributions}

JFH designed the study and acquired the data. All authors were involved in data analysis and interpretation. All authors contributed to the drafting and critical review of the manuscript and approved the final draft.

\section{Funding}

This study was supported by grant 211085/Z/18/Z from the Wellcome Trust (JFH), grant 101143/Z/13/Z from the Wellcome Trust (LT), grant MR/N013867/ 1 from the MRC (EF), NIHR UCLH Biomedical Research Centre (JFH, DPJO, GA, GL), NIHR ARC North Thames (JFH, DPJO), NIHR Oxford Health Biomedical Research Centre (JRG), Research Grants Council Hong Kong (ICKW). The funders were not involved in the study design, data collection, analysis, interpretation, report writing or submission.

\section{Availability of data and materials}

The anonymized patient-level data used for this project cannot be shared for reasons of information governance. However, data can be obtained by application to Clinical Practice Research Datalink. Code lists are available from the corresponding author on request.

\section{Declarations}

\section{Ethics approval and consent to participate}

Ethical approval for this study was obtained from the Independent Scientific Advisory Committee of CPRD (protocol no. 18_316). Informed consent was waived because data are anonymised for research purposes.

\section{Consent for publication}

Not applicable.

\section{Competing interests}

ICKW received research funding from Innovative Medicines Initiative, Shire, Janssen-Cilag, Eli-Lily, Pfizer, Bayer, Amgen outside the submitted work. No other authors declare competing interests.

\footnotetext{
Author details

${ }^{1}$ Division of Psychiatry, UCL, London, UK. ${ }^{2}$ Camden and Islington NHS Foundation Trust, London, UK. ${ }^{3}$ Department of Statistical Science, UCL, London, UK. ${ }^{4}$ Department of non-Communicable Disease Epidemiology, LSHTM, London, UK. 'Division of Software and Computer Systems, School of Electrical Engineering and Computer Science KTH, Stockholm, Sweden. ${ }^{6}$ Department of Learning, Informatics, Management and Ethics, Karolinska Institute, Solna, Sweden. ${ }^{7}$ Centre for Safe Medication Practice and Research, Department of Pharmacology and Pharmacy, The University of Hong Kong, Pokfulam, Hong Kong. ${ }^{8}$ Research Department of Practice and Policy, School of Pharmacy, UCL, London, UK. 'Department of Psychiatry, University of
}

Oxford, Oxford, UK. ${ }^{10}$ Department of Global Public Health, Karolinska Institute, Stockholm, Sweden.

Received: 5 January 2021 Accepted: 17 March 2021

Published online: 28 April 2021

\section{References}

1. Barroilhet SA, Ghaemi SN. When and how to use lithium. Acta Psychiatrica Scandinavica. 2020;142(3):161-72.

2. Tondo L, Alda M, Bauer M, Bergink V, Grof P, Hajek T, et al. Clinical use of lithium salts: quide for users and prescribers. Int J Bipolar Disorders. 2019; 7(1):16. https://doi.org/10.1186/s40345-019-0151-2.

3. Gitlin M. Lithium side effects and toxicity: prevalence and management strategies. Int J Bipolar Disorders. 2016;4(1):1-10.

4. McKnight RF, Adida M, Budge K, Stockton S, Goodwin GM, Geddes JR. Lithium toxicity profile: a systematic review and meta-analysis. Lancet. 2012; 379(9817):721-8. https://doi.org/10.1016/S0140-6736(11)61516-X.

5. Hayes JF, Marston L, Walters K, Geddes JR, King M, Osborn DP. Adverse renal, endocrine, hepatic, and metabolic events during maintenance mood stabilizer treatment for bipolar disorder: a population-based cohort study. PLOS medicine. 2016;13(8):e1002058.

6. Iwagami M, Mansfield KE, Hayes JF, Walters K, Osborn DP, Smeeth L, Nitsch D, Tomlinson L. Severe mental illness and chronic kidney disease: a crosssectional study in the United Kingdom. Clin Epidemiol. 2018;10:421-9. https://doi.org/10.2147/CLEP.S154841.

7. Kessing LV, Gerds TA, Feldt-Rasmussen B, Andersen PK, Licht RW. Use of lithium and anticonvulsants and the rate of chronic kidney disease: a nationwide population-based study. JAMA Psychiatry. 2015;72(12):1182-91. https://doi.org/10.1001/jamapsychiatry.2015.1834.

8. Gupta S, Khastgir U. Drug information update. Lithium and chronic kidney disease: debates and dilemmas. BJPsych Bull. 2017;41(4):216-20. https://doi. org/10.1192/pb.bp.116.054031.

9. Kazancioğlu R. Risk factors for chronic kidney disease: an update. Kidney Int Suppl. 2013;3(4):368-71. https://doi.org/10.1038/kisup.2013.79.

10. Lim CC, Chee ML, Cheng C-Y, Kwek JL, Foo M, Wong TY, Sabanayagam C. Simplified end stage renal failure risk prediction model for the low-risk general population with chronic kidney disease. PLoS One. 2019;14(2): e0212590. https://doi.org/10.1371/journal.pone.0212590.

11. Ramspek CL, de Jong Y, Dekker FW, van Diepen M. Towards the best kidney failure prediction tool: a systematic review and selection aid. Nephrology Dialysis Transplantation. 2020;35(9):1527-38.

12. Johnson ES, Thorp ML, Platt RW, Smith DH. Predicting the risk of dialysis and transplant among patients with CKD: a retrospective cohort study. Am J Kidney Dis. 2008;52(4):653-60. https://doi.org/10.1053/j.ajkd.2008.04.026.

13. Rigatto C, Sood MM, Tangri N. Risk prediction in chronic kidney disease: pitfalls and caveats. Curr Opin Nephrol Hypertens. 2012;21(6):612-8. https:// doi.org/10.1097/MNH.0b013e328359072f.

14. Tangri N, Grams ME, Levey AS, Coresh J, Appel LJ, Astor BC, Chodick G, Collins AJ, Djurdjev O, Elley CR, Evans M, Garg AX, Hallan SI, Inker LA, Ito S, Jee $\mathrm{SH}$, Kovesdy CP, Kronenberg F, Heerspink HJL, Marks A, Nadkarni GN, Navaneethan SD, Nelson RG, Titze S, Sarnak MJ, Stengel B, Woodward M, Iseki K, for the CKD Prognosis Consortium. Multinational assessment of accuracy of equations for predicting risk of kidney failure: a meta-analysis. Jama. 2016;315(2):164-74. https://doi.org/10.1001/jama.2015.18202.

15. Herrett E, Gallagher AM, Bhaskaran K, Forbes H, Mathur R, Van Staa T, et al. Data resource profile: clinical practice research datalink (CPRD). Int J Epidemiol. 2015:44(3):827-36. https://doi.org/10.1093/ije/dyv098.

16. Wolf A, Dedman D, Campbell J, Booth H, Lunn D, Chapman J, et al. Data resource profile: Clinical practice research datalink (cprd) aurum. Int J Epidemiol. 2019;48(6):1740-g.

17. Matsushita K, Mahmoodi BK, Woodward M, Emberson JR, Jafar TH, Jee SH, Polkinghorne KR, Shankar A, Smith DH, Tonelli M, Warnock DG, Wen CP, Coresh J, Gansevoort RT, Hemmelgarn BR, Levey AS, Chronic Kidney Disease Prognosis Consortium. Comparison of risk prediction using the CKD-EPI equation and the MDRD study equation for estimated glomerular filtration rate. Jama. 2012;307(18):1941-51. https://doi.org/10.1001/jama.2012.3954.

18. Nagin DS, Odgers CL. Group-based trajectory modeling in clinical research. Annu Rev Clin Psychol. 2010;6(1):109-38. https://doi.org/10.1146/annurev. clinpsy.121208.131413.

19. Nylund $\mathrm{KL}$, Asparouhov T, Muthén BO. Deciding on the number of classes in latent class analysis and growth mixture modeling: a Monte Carlo 
simulation study. Struct Equ Model Multidiscip J. 2007;14(4):535-69. https:// doi.org/10.1080/10705510701575396.

20. Davis J, Desmond M, Berk M. Lithium and nephrotoxicity: a literature review of approaches to clinical management and risk stratification. BMC Nephrol. 2018;19(1):1-7.

21. Zou H, Hastie T. Regularization and variable selection via the elastic net. J Royal Statistical Society: Series B (Stat Methodol). 2005;67(2):301-20. https:// doi.org/10.1111/j.1467-9868.2005.00503.x.

22. Tangri N, Stevens LA, Griffith J, Tighiouart H, Djurdjev O, Naimark D, Levin A, Levey AS. A predictive model for progression of chronic kidney disease to kidney failure. Jama. 2011;305(15):1553-9. https://doi. org/10.1001/jama.2011.451.

23. Nattino G, Finazzi S, Bertolini G. A new calibration test and a reappraisal of the calibration belt for the assessment of prediction models based on dichotomous outcomes. Stat Med. 2014;33(14):2390-407. https://doi.org/1 $0.1002 / \operatorname{sim} .6100$

24. StataCorp L. Stata statistical software: release 16. TX: College Station; 2019.

25. Carrero JJ, Hecking M, Chesnaye NC, Jager KJ. Sex and gender disparities in the epidemiology and outcomes of chronic kidney disease. Nat Rev Nephrol. 2018;14(3):151-64. https://doi.org/10.1038/nrneph.2017.181.

26. Shine B, McKnight RF, Leaver L, Geddes JR. Long-term effects of lithium on renal, thyroid, and parathyroid function: a retrospective analysis of laboratory data. Lancet. 2015;386(9992):461-8. https://doi.org/10.1016/5014 0-6736(14)61842-0

27. Major RW, Shepherd D, Medcalf JF, Xu G, Gray LJ, Brunskill NJ. The kidney failure risk equation for prediction of end stage renal disease in UK primary care: an external validation and clinical impact projection cohort study. PLoS Med. 2019;16(11):e1002955. https://doi.org/10.1371/ journal.pmed.1002955

28. Weng S-C, Wu C-L, Kor C-T, Chiu P-F, Wu M-J, Chang C-C, Tarng DC Migraine and subsequent chronic kidney disease risk: a nationwide population-based cohort study. BMJ Open. 2017;7(12):e018483. https://doi. org/10.1136/bmjopen-2017-018483.

29. Leo RJ, Singh J. Migraine headache and bipolar disorder comorbidity: a systematic review of the literature and clinical implications. Scand J Pain. 2016;11(1):136-45. https://doi.org/10.1016/j.spain.2015.12.002.

\section{Publisher's Note}

Springer Nature remains neutral with regard to jurisdictional claims in published maps and institutional affiliations.

Ready to submit your research? Choose BMC and benefit from:

- fast, convenient online submission

- thorough peer review by experienced researchers in your field

- rapid publication on acceptance

- support for research data, including large and complex data types

- gold Open Access which fosters wider collaboration and increased citations

- maximum visibility for your research: over $100 \mathrm{M}$ website views per year

At $\mathrm{BMC}$, research is always in progress.

Learn more biomedcentral.com/submissions 